\title{
A General Relation Between Membrane Potential, Ion Activities, and Pump Fluxes for Symmetric Cells in a Steady State
}

\author{
JOHN A. JACQUEZ \\ Department of Physiology, The University of Michigan, Medical School, \\ Ann Arbor, Michigan 48104 \\ AND \\ STANLEY G. SCHULTZ \\ Department of Physiology, University of Pittsburgh, School of Medicine, \\ Pittsburgh, Pennsylvania 15213
}

\begin{abstract}
For steady states of cells that have uniform cell membranes, there must be zero net fluxes of each ion. This provides a set of conditions more restrictive than the condition of zero net current flow that is used to derive the Goldman equation. We show that this leads to a set of general relations between the membrane potential, ion activities, ion permeabilities, and pump fluxes and that the Goldman equation is but one of this set. Further, we demonstrate that the transmembrane potential is uniquely defined by the intracellular and extracellular activities, the permeability coefficients, and the ratio of the net mediated (nondiffusional) fluxes of any two ions of the same valence in a steady state, regardless of the behavior of other ions and without assumptions with respect to the electrical potential profile across the membrane. In the course of this, we also give an exact and general derivation of the Mullins-Noda relation.
\end{abstract}

\section{INTRODUCTION}

The Goldman equation as originally derived $[1,2]$ depended on the assumptions of a constant field across the membrane, zero net current flow across the membrane, and zero net current due to pumps. Over the years efforts to generalize the Goldman equation have been directed to relaxing the constant field assumption [3-7] and to incorporating the effects of electrogenic pumps [6-12]. General derivations that do both are now available $[6,7]$.

On reviewing the applicability of the Goldman equation, one of us (S.G.S.) noted that for a steady state distribution of ions in symmetric cells 
that have uniform membrane properties, many more restrictions on fluxes hold than are required for the derivation of the generalized Goldman equation $[6,7]$. This raised the possibility that relations other than the Goldman equation connecting ion fluxes and the membrane potential might be found. The basic assumption used to derive the generalized Goldman equation is that there is a zero net rate of charge transfer across the membrane. This is given by

$$
\sum_{k} z_{k}\left[J_{k}^{d}+J_{k}^{p}\right]=0,
$$

in which $z_{k}, J_{k}^{d}$, and $J_{k}^{p}$ are the valence, net diffusive, and net mediated fluxes of the $k$ th ion. We use the convention that fluxes are positive when directed into the cell. We also note that the derivation of the generalized Goldman equation depends on the assumption that only univalent ions are present. Equation (1) introduces no assumption of a steady state for any ion; all it does is to impose a particular restriction on total current flow. However, for a symmetric cell that has uniform membrane properties, the steady state condition implies there is a zero net flux for each ion, as given by Eq. (2), which is much more restrictive than Eq. (1).

$$
J_{k}^{d}+J_{k}^{p}=0 .
$$

Note that we have excluded polarized cells in cellular membranes, which have at least two different membrane areas, apical and basal, that may have different potential profiles and non-zero net fluxes across each. For symmetric cells for which Eq. (2) holds it follows that Eq. (1) also holds, so the generalized Goldman equation (7) must also hold. However, since Eq. (2) is true for each ion, it must follow that Eq. (3) also holds, but now the $m_{k}$ are arbitrary multipliers.

$$
\sum_{k} m_{k}\left(J_{k}^{d}+J_{k}^{p}\right)=0 .
$$

This suggests that we may be able to find a more general class of relations, similar in some ways to the Goldman equation, and that the Goldman equation is but one of this set. In what follows we derive such a general relation that holds for steady states of cells with uniform membranes and for arbitrary potential profiles, and then show that if the mediated fluxes are known this leads to a set of useful relations between the membrane potential, the activities of the ions, the permeabilities, and the pump fluxes of the ions. 


\section{THEORY AND RESULTS}

In a previous paper [7] one of us has shown that for a steady state and arbitrary potential profile the diffusive flux of an ion across the cell membrane is given by Eq. (4), which is obtained by integrating the NernstPlanck equation across the cell membrane.

$$
J_{k}^{d}=\frac{-a P_{k}}{\int_{-a / 2}^{a / 2} \exp \left(z_{k} F \varphi / R T\right) d x}\left[c_{k}^{i} Z_{i}^{z_{k}} \exp \left(\frac{z_{k} F V}{2 R T}\right)-c_{k}^{o} Z_{0}^{-z_{k}} \exp \left(-\frac{z_{k} F V}{2 R T}\right)\right]
$$

The center of the membrane is taken as origin of coordinates for a membrane of thickness $a$. In Eq. (4) $P_{k}$ is the permeability of the $k$ th ion, $z_{k}$ is its charge, $\varphi(x)$ is the potential at point $x$ in the membrane, $V$ is the potential difference across the membrane, $\varphi(a / 2)-\varphi(-a / 2)$, from the outside at $-a / 2$ to the inside at $a / 2, c_{k}$ is the activity of the $k$ th ion, and the superscripts $i$ and $o$ denote the intracellular and extracellular compartments respectively. The $Z$ factors are defined by $Z_{i}=\exp \left(F V_{i} / R T\right), Z_{o}$ $=\exp \left(F V_{o} / R T\right)$, where $V_{i}=\varphi_{i}-\varphi(a / 2)$ and $V_{o}=\varphi(-a / 2)-\varphi_{o}$ take into account the potential differences between inner and outer bulk phases and the adjacent membrane surfaces. The measured membrane potential is the difference in potential between inner and outer bulk phases, $V_{m}=\varphi_{i}-\varphi_{o}$ $=V_{i}+V+V_{v}$. We consider only univalent ions at first and generalize the result in the conclusion. Using the fact that the potential function can always be written as a sum of an even function, $\sigma(x)$, and an odd function, $\theta(x)$, i.e., $\varphi(x)=\sigma(x)+\theta(x)$, the relations (5) and (6) can be derived [7].

$$
\begin{array}{r}
\int_{-a / 2}^{a / 2} \exp (F \sigma / R T) \exp (F \theta / R T) d x=g \Omega=g \int_{-a / 2}^{a / 2} \exp (F \theta / R T) d x \\
\int_{-a / 2}^{a / 2} \exp (-F \sigma / R T) \exp (-F \theta / R T) d x=h \Omega=h \int_{-a / 2}^{a / 2} \exp (F \theta / R T) d x
\end{array}
$$

The factors $g$ and $h$ are mean values across the membrane of $\exp (F \sigma(x) /$ $R T)$ and $\exp (-F \sigma(x) / R T)$ respectively with respect to the weighting function $\exp (F \theta(x) / R T)$. If these are substituted in Eq. (4), we obtain Eqs. (7) and (8), where the subscripts $k$ and $j$ now denote univalent positive and negative ions respectively.

$$
J_{k}^{d} \frac{\Omega}{a}=-\frac{P_{k}}{g}\left[c_{k}^{i} Z_{i} \exp \left(\frac{F V}{2 R T}\right)-c_{k}^{o} Z_{o}^{-1} \exp \left(-\frac{F V}{2 R T}\right)\right]
$$




$$
J_{j}^{d} \frac{\Omega}{a}=-\frac{P_{j}}{h}\left[c_{j}^{i} Z_{i}^{-1} \exp \left(-\frac{F V}{2 R T}\right)-c_{j}^{o} Z_{o} \exp \left(\frac{F V}{2 R T}\right)\right]
$$

Now we consider a steady state in which the net flux of each of some subset of ions is zero. This need not be true for all ions, only for those we use in the following derivation, but the cell must be in a steady state in which the potential profile does not change with time. We use $J_{k}^{p}$ to represent net mediated flux (i.e. non-diffusive) of the $k$ th ion and again use $k$ and $j$ as subscripts for positive and negative ions respectively. It should be emphasized that $J_{k}^{p}$ is not just $a$ pump flux, it is the sum of all fluxes due to mechanisms other than diffusion of free ion $k$ and could include fluxes due to cotransport of the ion with other substrates. Then Eqs. (9) and (10) hold for the positive and negative univalent ions respectively.

$$
\begin{aligned}
& J_{k}^{d}+J_{k}^{p}=0 \\
& J_{j}^{d}+J_{j}^{p}=0
\end{aligned}
$$

Ions that are at electrochemical equilibrium need not be considered. If (9) and (10) hold, then any linear combination of such equations is also zero, from which one obtains

$$
\sum_{k} m_{k} J_{k}^{d}+\sum_{j} n_{j} J_{j}^{d}=-\left[\sum_{k} m_{k} J_{k}^{p}+\sum_{j} n_{j} J_{j}^{p}\right]
$$

in which $m_{k}$ and $n_{j}$ are arbitrary coefficients which we will take to be real numbers. Further, if either the diffusional fluxes or the pump fluxes of the ions are known, one can choose $m_{k}$ and $n_{j}$ such that both sides of Eq. (11) equal zero. Obviously Eq. (11) holds for any subset of ions for which Eqs. (9) and (10) hold. If we multiply Eq. (11) by $\Omega / a$ and substitute for the diffusive fluxes from Eqs. (7) and (8), Eq. (12) is obtained after some algebraic manipulation which follows the pattern used in a previous paper $[7]$.

$$
V_{m}=\frac{R T}{F} \ln \left\{\begin{array}{c}
\sum_{k} m_{k} \frac{P_{k}}{g} c_{k}^{o}-\sum_{j} n_{j} \frac{P_{j}}{h} Z_{o} Z_{i}^{-1} c_{j}^{i} \\
+\frac{\Omega}{a} Z_{o} \exp \left(\frac{F V}{2 R T}\right)\left[\sum_{k} m_{k} J_{k}^{p}+\sum_{j} n_{j} J_{j}^{p}\right]
\end{array}\right\}
$$


This is the general result for univalent ions.

Although of theoretical interest, the usefulness of Eq. (12) is impaired by the presence of the unknown integrals $\Omega \exp (F V / 2 R T)$ in the numerator. Note that $P_{k} / g$ and $P_{j} / h$ are apparent permeabilities for the particular steady state involved, so $g$ and $h$ are not so bothersome as is the presence of the last term in the numerator. However, the $m_{k}$ and $n_{j}$ are arbitrary, and if the number of ions involved is two or more and if we know the pump fluxes, then there are many choices of the $m_{k}$ and $n_{j}$ that make the linear combination of pump fluxes in the last term in the numerator disappear. This, of course, defines the $m_{k}$ and $n_{j}$ in terms of the pump fluxes through the linear relations

$$
\sum_{k} m_{k} J_{k}^{p}+\sum_{j} n_{j} J_{j}^{p}=0
$$

Much more than this can be asserted. Since we can form Eq. (11) for any subset of univalent ions for which Eqs. (9) and (10) hold, there is an equation of the form of (12) for each ion, for every pair of pumped univalent ions, for every triple, and so forth. The equation for a single ion is obtainable fairly directly from (7) and (9). It appears to be of little practical value because there is no way to eliminate the unknown integrals $\Omega$ and $\exp (F V / 2 R T)$. The other equations are more interesting. If we consider any pair of pumped ions, say $k=1, j=1$, then the relation (13) shows that $m_{1} / n_{1}$ is a ratio of pump fluxes. It is especially useful to choose a pair of ions of the same sign, such as sodium and potassium, which are of major importance in cellular physiology.

For sodium and potassium Eq. (12) reduces to

$V_{m}=R T \operatorname{F} \ln \left\{\begin{array}{c}m_{\mathrm{Na}} P_{\mathrm{Na}} c_{\mathrm{Na}}^{o}+m_{\mathrm{K}} P_{\mathrm{K}} c_{\mathrm{K}}^{o} \\ +\left[g \Omega Z_{o} \exp (F V / 2 R T) / a\right]\left[m_{\mathrm{Na}} J_{\mathrm{Na}}^{p}+m_{\mathrm{K}} J_{\mathrm{K}}^{p}\right]\end{array}\right\}$

If we know the ratio of total mediated fluxes for sodium and potassium, we simply choose $m_{\mathrm{Na}}$ and $m_{\mathrm{K}}$ to satisfy

$$
m_{\mathrm{Na}} J_{\mathrm{Na}}^{p}+m_{\mathrm{K}} J_{\mathrm{K}}^{p}=0
$$

Note again that $J_{\mathrm{Na}}^{p}$ and $J_{\mathrm{K}}^{p}$ are net mediated fluxes for each ion, not just linked $\mathrm{Na}^{+}-\mathrm{K}^{+}$pump fluxes. Substitution of (15) into (14) gives

$$
V_{m}=\frac{R T}{F} \ln \left\{\frac{c_{\mathrm{K}}^{o}-\frac{J_{\mathrm{K}}^{p}}{J_{\mathrm{Na}}^{p}} \frac{P_{\mathrm{Na}}}{P_{\mathrm{K}}} c_{\mathrm{Na}}^{o}}{c_{\mathrm{K}}^{i}-\frac{J_{\mathrm{K}}^{p}}{J_{\mathrm{Na}}^{p}} \frac{P_{\mathrm{Na}}}{P_{\mathrm{K}}} c_{\mathrm{Na}}^{i}}\right\} .
$$


For the particular case that the mediated fluxes of sodium and potassium are equal and opposite, $J_{\mathrm{K}}^{p} / J_{\mathrm{Na}}^{p}=-1$, and Eq. (16) reduces to what appears to be a special case of the Goldman equation. Equation (16) was derived by Mullins and Noda [9] assuming that the cell was in a steady state, that only $\mathrm{Na}^{+}$and $\mathrm{K}^{+}$were pumped, and that $\mathrm{Cl}^{-}$was distributed at electrochemical equilibrium. The present derivation is less restricted inasmuch as it demonstrates that the membrane potential is uniquely determined by the intracellular and extracellular activities, the permeability coefficients, and the ratio of the net mediated pump fluxes of any two ions in a steady state regardless of whether other ions, univalent or multivalent, are pumped.

It is possible to include ions of any charge in the derivation, but then a number of different unknown integrals of the type of $\Omega$ appear on the left side in the equations equivalent to Eqs. (7) and (8), and the results are not useful. However, a useful result is obtained if one focuses on pumped ions of one absolute valence. Now let $z$ be the absolute value of the valence. We again use subscript $k$ for the positive ions of valence $+z$, and $j$ for the negative ions of valence $-z$. Then the generalization of Eq. (12) for ions of one absolute valence, $z$, is

$$
V_{m}=\frac{R T}{z F} \ln \left\{\begin{array}{r}
\sum_{k} m_{k} \frac{P_{k}}{G} c_{k}^{o}-\sum_{j} n_{j} \frac{P_{j}}{H} c_{j}^{i} Z_{o}^{z} Z_{i}^{-z} \\
+\frac{\Gamma}{a} Z_{o}^{z} \exp \left(\frac{z F V}{2 R T}\right)\left[\sum_{k} m_{k} J_{k}^{p}+\sum n_{j} J_{j}^{p}\right] \\
\sum m_{k} \frac{P_{k}}{G} c_{k}^{i}-\sum_{j} n_{j} \frac{P_{j}}{H} c_{j}^{o} Z_{o}^{z} Z_{i}^{-z}
\end{array}\right\}
$$

In Eq. (17) $\Gamma$ is the integral of $\exp (z F \theta / R T)$ across the membrane, and $G$ and $H$ are defined as the mean values of $\exp (z F \sigma / R T)$ and $\exp (-z F \sigma /$ $R T$ ) with respect to $\exp (z F \theta / R T)$. One can now look at particular cases, as we did for univalent ions. Thus, if calcium and magnesium were both transported by a particular cell, one would obtain

$$
V_{m}=\frac{R T}{2 F} \ln \left\{\frac{c_{\mathrm{Ca}}^{o}-\frac{J_{\mathrm{Ca}}^{p}}{J_{\mathrm{Mg}}^{p}} \frac{P_{\mathrm{Mg}}}{P_{\mathrm{Ca}}} c_{\mathrm{Mg}}^{o}}{c_{\mathrm{Ca}}^{i}-\frac{J_{\mathrm{Ca}}^{p}}{J_{\mathrm{Mg}}^{p}} \frac{P_{\mathrm{Mg}}}{P_{\mathrm{Ca}}} c_{\mathrm{Mg}}^{i}}\right\}
$$

This work was supported in part by grants from the National Cancer Institute (CA-06734), the National Institute of General Medical Sciences (GM-00892), the National Institute of Arthritis, Metabolism and Digestive Diseases (AM-16275), and the American Heart Association (74-684). 


\section{REFERENCES}

1 D. W. Goldman, J. Gen. Physiol. 27, 37 (1943).

2 A. L. Hodgkin and B. Katz, J. Physiol. (London) 108, 37 (1949).

3 L. Barr, J. Theoret. Biol. 19, 67 (1965).

4 J. P. Sandblom and G. Eisenman, Biophys. J. 7, 217 (1967).

5 A. D. MacGillivray and D. Hare, J. Theoret. Biol. 25, 113 (1969).

6 T. L. Schwartz, Biophys. J. 11, 944 (1971).

7 J. A. Jacquez, Math. Biosci. 12, 185 (1971).

8 G. E. Briggs, Proc: Roy, Soc. (London) B 156, 573 (1962).

9 L. J. Mullins and K. Noda, J. Gen. Physiol. 47, 117 (1963).

10 D. Geduldig, J. Theoret. Biol. 19, 67 (1968).

11 R. B. Moreton, J. Exp. Biol. 51, 181 (1969).

12 M. F. Marmor, J. Physiol. (London) 218, 599 (1971). 